\title{
Ein byzantinisches Schulgespräch.
}

C. Wachsmuth brachte im 34. Bande des Rheinischen Museums S. 156 ein handschriftliches Zeugnis, welches zeigt, wie wohl bekannt den späteren Byzantinern Heliodoros als einer der hauptsächlichsten Commentatoren der Dionysischen Techne gewesen ist. Dasselbe ist dem Anfange eines im cod. Vallicellianus F 68 (V) befindlichen Schulgespräches entnommen. Eben dieses Schulgespräch habe ich vor fünf Jahren in Mailand aus dem cod. Ambrosianus C 222 inf. (A), fol. 338 ${ }^{\mathrm{rv}}$, abgeschrieben. Ich that das auf Anregung Wilhelm Studemunds. Er gab mir damals die Notizen, welche er sich am 1. Oktober 1883 über jene Seiten von A gemacht und die er dem Berichte in seinen Anecdota Graeca, Berlin 1886, S. 247, zu Grunde gelegt. Als ich mir 1889 eine vollständige Abschrift des Schulgespräches auch aus $V$ verschaffen wollte, schrieb mir A. Mau, das Blatt, auf welchem es gestanden, sei verschwunden; es müsse vor der 1884 erfolgten Nummerierung der Blätter der Handschrift verloren gegangen sein. Vor zwei Jahren habe ich mich selbst davon überzeugt: das Blatt vor Fol. 198 in $V$ fehlt, auf Fol. $198^{\mathrm{r}}$ stehen nur noch die letzten Zeilen des Gespräches von $\mu \varepsilon-$ $\mu \alpha^{\prime} \vartheta \eta x \alpha S$ in der Zeile 88 bis zum Schlufs; alles übrige fehlt. Doch dieser Verlust ist zu verschmerzen; denn Herr Geheimrat C. Wach smuth hatte die grofse Freundlichkeit mir von einer in seinem Besitz befindlichen sehr sorgfältigen, selbst die Züge der Handschrift nachmalenden Kopie von V, welche sein Schwiegervater Friedrich Ritschl gemacht hatte, die abweichenden Lesarten in meinen Text einzutragen. $\mathrm{V}$ ist ziemlich fehlerhaft geschrieben, hat mir aber doch für die richtige Lesung von A Dienste geleistet, denn die berühmte Handschrift A (aus dem Ende des 13. Jahrhunderts) ist bekanntlich im allgemeinen aufserordentlich schwer zu lesen; der Text ist auf weichem faserigen Bombycin geschrieben, die Schrift tachygraphisch und sehr häufig ganz verblalst. Im übrigen bemerke ich, dafs in A das $\iota$ subscriptum gewöhnlich fehlt,

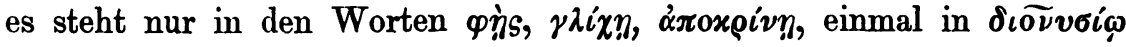
und $\pi \varepsilon i \rho \alpha$. Die Accente meines Textes sind die von $A$, in der Interpunktion weiche ich natürlich $\mathrm{ab}$; zu bemerken ist jedoch, dafs das 
Fragezeichen, wie so oft in Handschriften des 13. und 14. Jahrhunderts, immer nur nach denjenigen Fragen gesetzt ist, welche nicht durch Fragepartikeln oder Fragepronomina als solche zu erkennen sind.

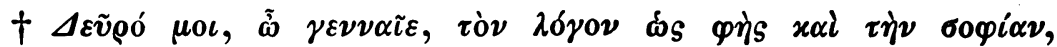

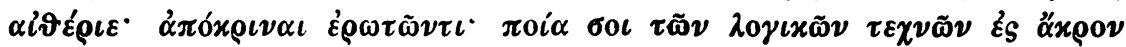

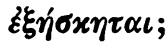

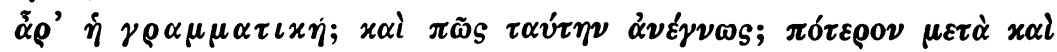

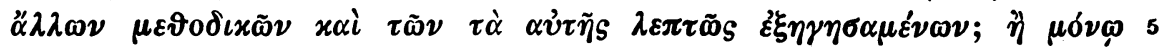

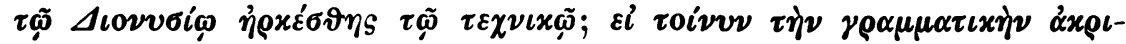

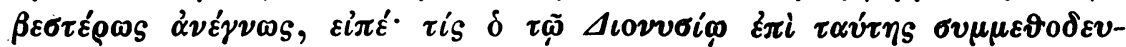

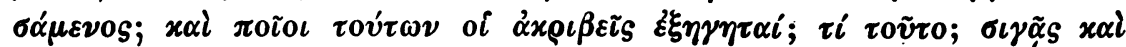

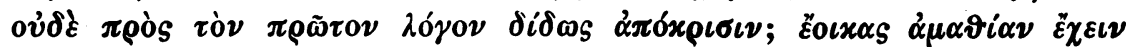

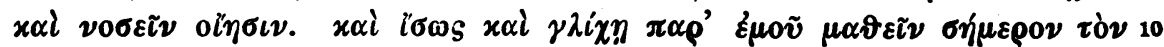

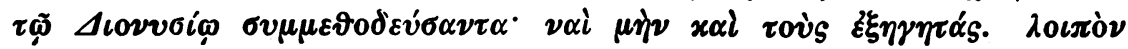

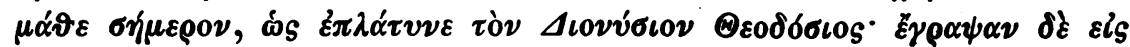

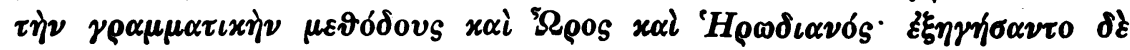

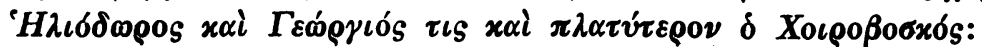

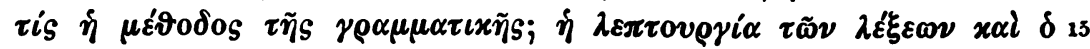

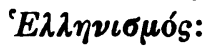

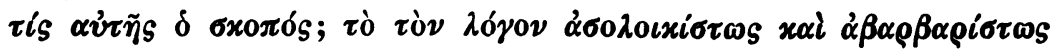
$\pi \rho \circ \varphi \varepsilon ́ \rho \varepsilon \sigma \vartheta \alpha \iota:$

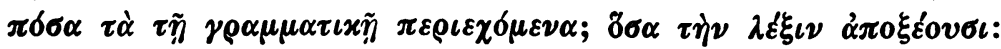

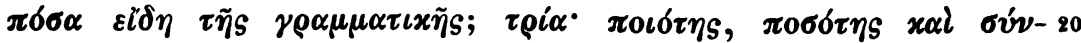
$\tau \alpha \xi \iota s:-$

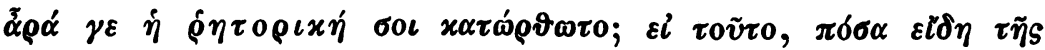

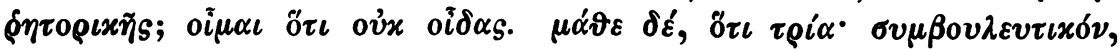

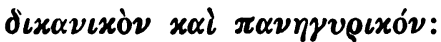

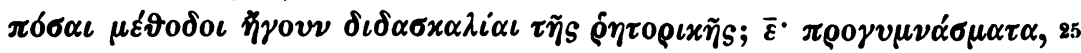

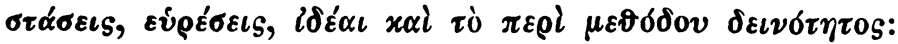

$\pi \delta \sigma \alpha \alpha \rho \sigma \gamma \mu \nu \alpha \dot{\sigma} \mu \alpha \tau \alpha ; \overline{\iota \delta}$ :

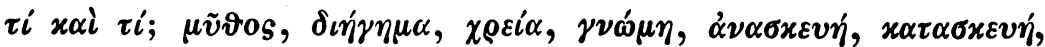

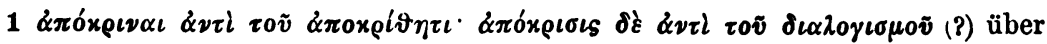

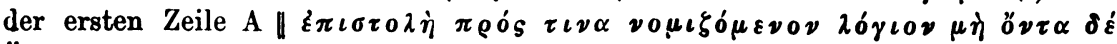

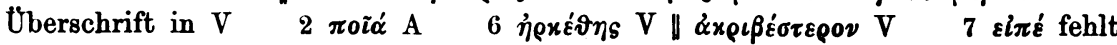

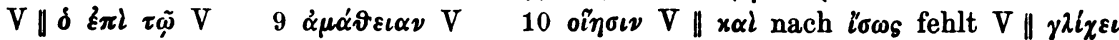

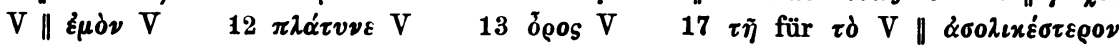

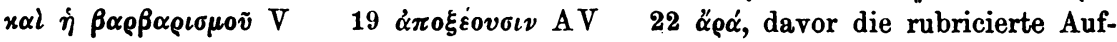

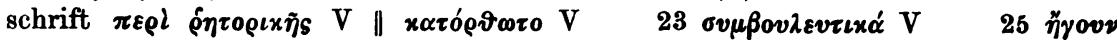
$\delta \omega \delta \alpha \delta \alpha \lambda l \alpha \iota$ von erster Hand über der Zeile A $28 \alpha^{\prime} \mu \tilde{v} \vartheta 0 \xi^{\cdot} \beta^{\prime} \delta \iota \eta^{\prime} \gamma \eta \mu \alpha$ und

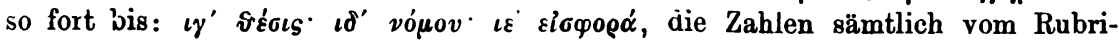
kator $\mathbf{V}$

Byzant. Zeitschrift II 1. 


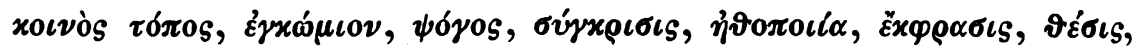

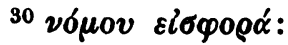

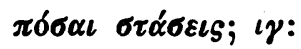

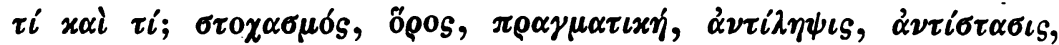

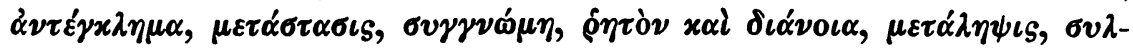
$\lambda о \gamma \iota \sigma \mu \delta ́ s, \dot{\alpha} \nu \tau \iota \nu 0 \mu i \alpha, \dot{\alpha} \mu \varphi \iota \beta 0 \lambda i \alpha$ :

35

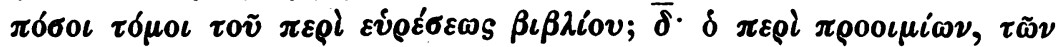

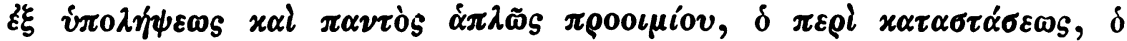

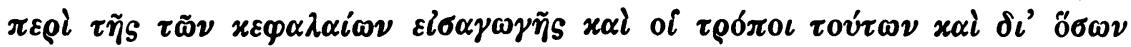

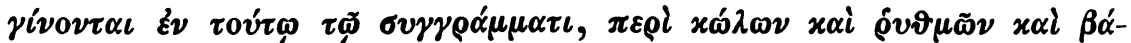

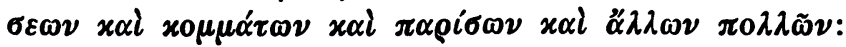

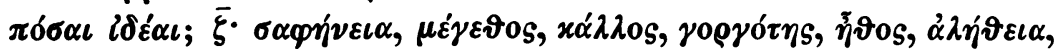
$\delta \varepsilon \iota \nu o ́ \tau \eta s:$

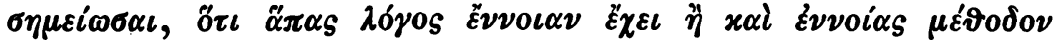

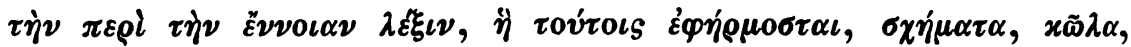

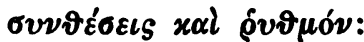

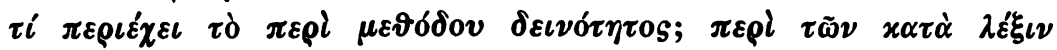

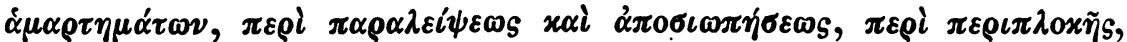

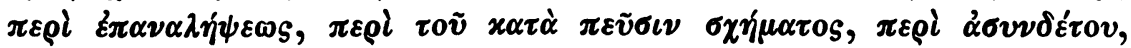

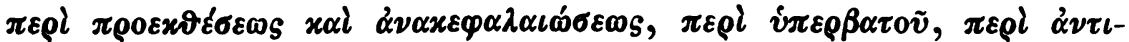

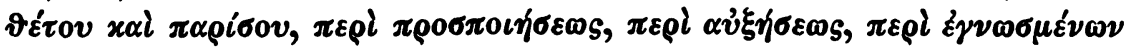

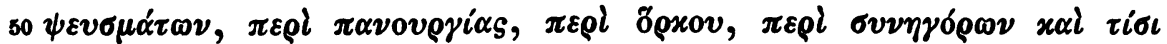

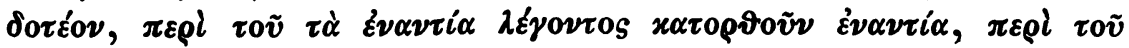

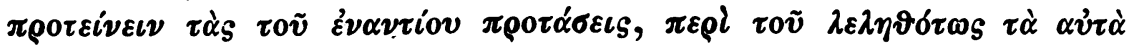

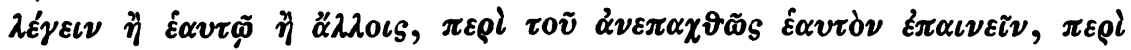

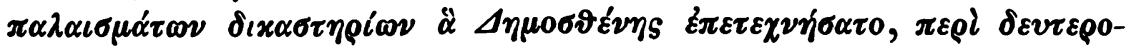

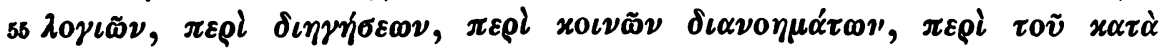

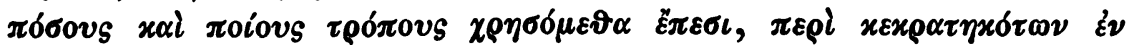

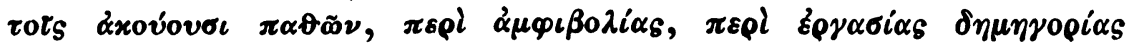

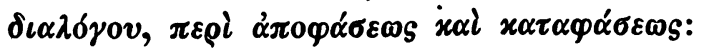

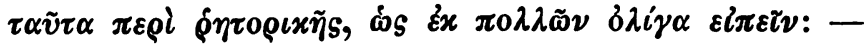

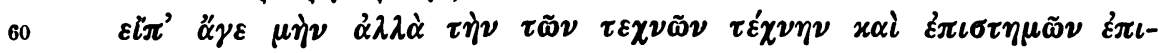

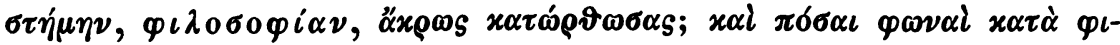

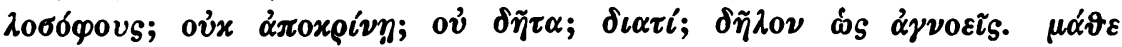

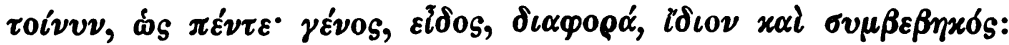

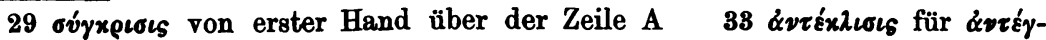

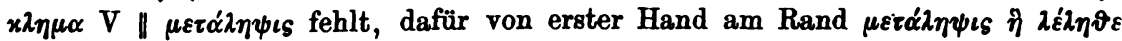

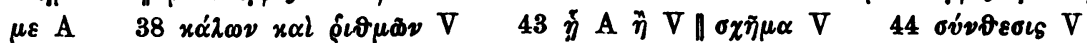

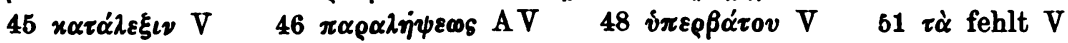

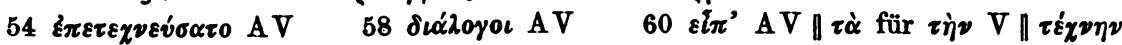

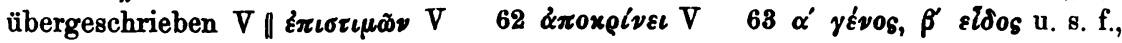
auch hier die Zahlen vom Rubrikator $\mathrm{V}$ 


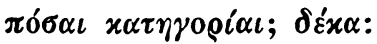

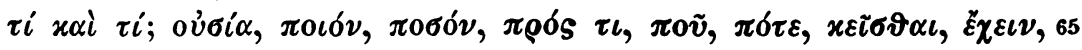
$\pi 0 \iota \varepsilon i \nu, \pi \alpha^{\prime} \sigma \chi \varepsilon \iota \nu$ :

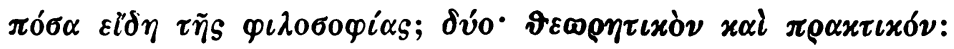

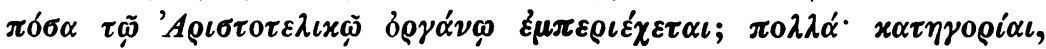

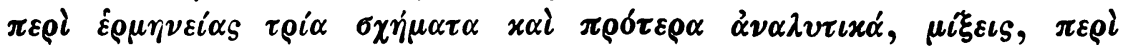

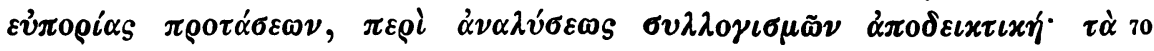

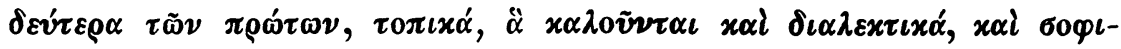

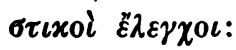

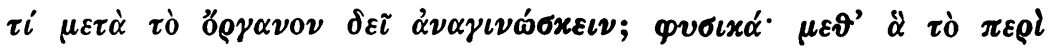

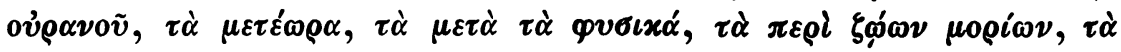

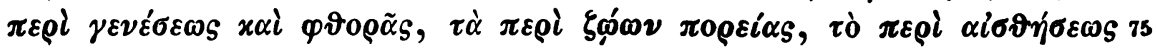

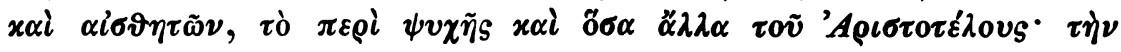

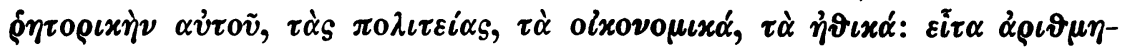

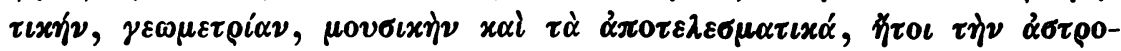

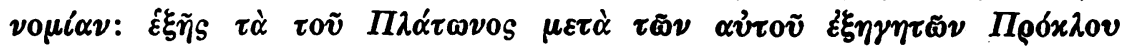

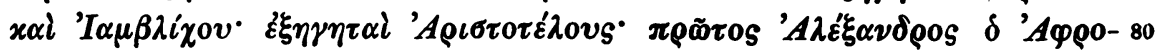

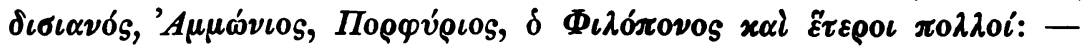

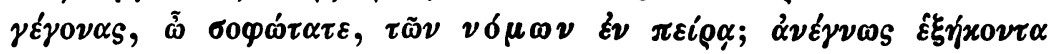

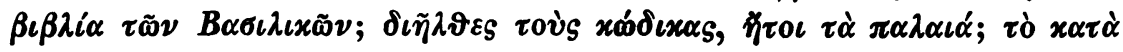

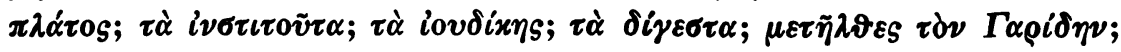

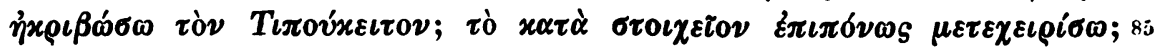

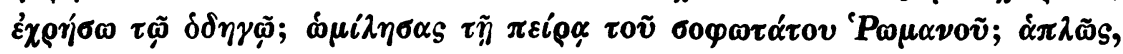

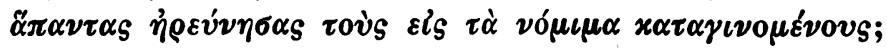

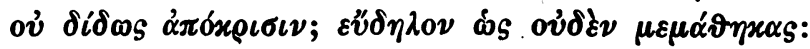

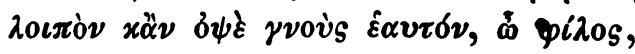

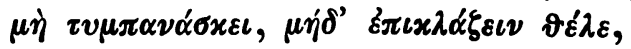

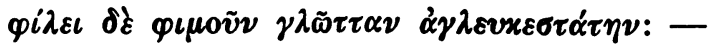

Der Schreiber von V sah in dem vorstehenden Schriftstück einen Brief an jemand, der sich einbildete gebildet zu sein. Das ist natürlich nur ein seltsames Mifsverständnis. Aber auch die Bezeichnung desselben als eines colloquium scholasticum, eines Schulgespräches, ist mifslich, spricht ja doch nur der Lehrer allein. Mit anerkennenswerter Unverdrossenheit stellt er Fragen, aber ohne Erfolg: sein Schüler thut nicht ein einziges Mal den Mund auf. Und so giebt er denn, aller-

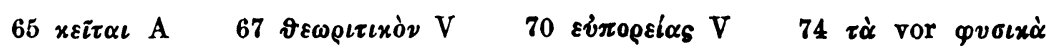

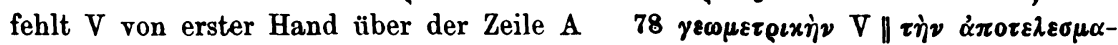

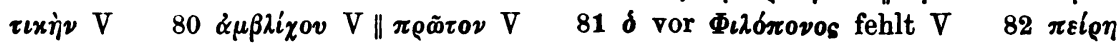

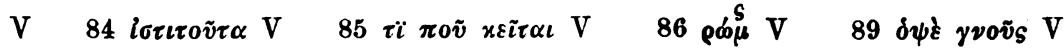
$91 \delta \dot{\varepsilon} \mathrm{V}$ 
dings nicht ohne dies beharrliche Schweigen wiederholt für einen Beweis bedauerlicher Unwissenheit zu erklären, alle Antworten selber.

In dieser etwas ungewöhnlichen Form der Belehrung erhalten wir nun eine Übersicht über alle diejenigen Wissensgebiete, welche nach der Meinung des Lehrers ein gebildeter Mann beherrschen mufs. Es sind das im ganzen vier: Grammatik, Rhetorik, Philosophie und Rechtskunde.

Die Auskunft aber, welche unser Magister über die Anforderungen giebt, welche er in jenen Wissenschaften erfüllt wissen will, ist freilich sonderbar genug. Sie erweckt in uns schwere Bedenken über seinen Wissensstand, über seine Lehrbefähigung und über die Klarheit seines Kopfes. Er mag ja vielerlei studiert haben - besonders die Schulweisheit der Rhetoren scheint ihm ganz geläufig zu sein -, aber er verrät nicht, dafs er ein erfahrener Schulmeister ist, welcher den redlichen Willen hat seinen Zögling über seine Lehrziele wirklich aufzuklären: er ist nichts weiter als ein geschwätziger alter Praktikus, der ganz plan- und gedankenlos der augenblicklichen Eingebung folgend mit hochklingenden Namen und Begriffen um sich wirft. An wirklicher Belehrung ist ihm augenscheinlich nicht das geringste gelegen; es macht vielmehr ganz den Eindruck, als lege er es nur darauf an seinem unglücklichen Neuling mit einem Wust von Gelehrsamkeit zu imponieren und $\mathrm{ihm}$ das Studium gründlich $\mathrm{zu}$ verleiden: und diesen Zweck wird er ja wohl erreicht haben.

Aber leider ist dieses unsagbar klägliche Erzeugnis der Laune eines griesgrämigen Alten doch in mehrfacher Beziehung geeignet unser Interesse in Ansprach zu nehmen. Man wird es mir aber hoffentlich nicht verdenken, wenn ich keine besondere Neigung verspüre diese Lehrprobe meines byzantinischen Kollegen eingehender zu behandeln, sondern mich in wesentlichen darauf beschränke die Zeit der Abfassung dieses Gespräches zu bestimmen.

Georgios Akropolites, ohne Zweifel einer der vornehmsten Politiker und dazu einer der gebildetsten Männer des 13. Jahrhunderts, hat sich,

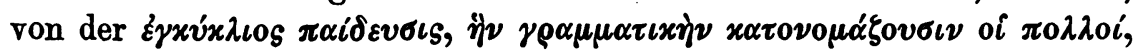
abgesehen, allein durch das Studium der Rhetorik und der Philosophie auf seine staatsmännische Laufbahn vorbereitet; sie beide allein ver-

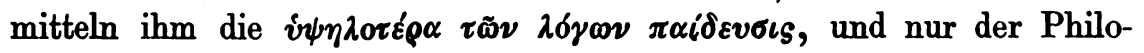
soph ist neben einem Könige nennenswert, wie Kaiser Johannes Dukas

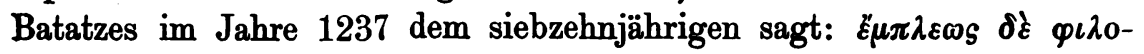

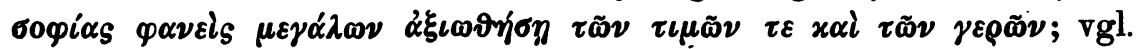
Geo. Akropolites ed. Bonn. p. 53, 16. Ganz in demselben Sinne spricht sich sein jüngerer Zeitgenosse und Freund Gregorios Kyprios, Patriarch 
von 1282-1289, in seiner bekannten Autobiographie und in seiner Lobschrift auf den Kaiser Michael Palaiologos aus. Rhetorik und Phi-

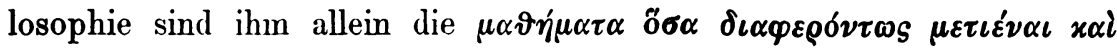

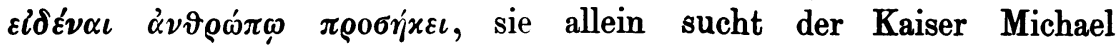
(1261-1282) in seiner wiedergewonnenen Hauptstadt durch sein $\mu \alpha \vartheta \eta$

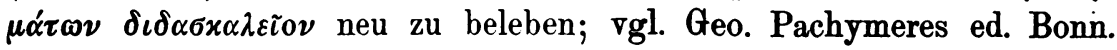
I 284. Der Begriff Philosophie ist natürlich in weiterem Sinne zu nehmen, er umfafste, wie wir das auch aus unserem Gespräche ersehen, nicht nur das Studium der Aristotelischen und Platonischen Schriften samt ihren Exegeten, sondern auch die Arithmetik, Geometrie, Musik und Astronomie. Aber dafs auch die Rechtskunde zu denjenigen Fächern gehöre, welche ein gebildeter Mann, zumal ein solcher, welcher sich dem Hof- und Staatsdienste widmen wollte, studiert haben müsse, das hat im 13. Jahrhundert zu Konstantinopel schwerlich jemand behauptet. Es ist bis jetzt nicht einmal nachgewiesen, dafs es in diesem Zeitraum im byzantinischen Reiche besondere Rechtsschulen gegeben hat. Ins 13. Jahrhundert, in dessen Ausgange unser Gespräch in der Handschrift A wahrscheinlich aufgeschrieben worden ist, werden wir also die Entstehung desselben nicht setzen dürfen.

Wie steht es mit dem 12. Jahrhundert? Theodoros Prodromos feiert seinen vornehmen Gönner, den Nomophylax, Protekdikos und Orphanotrophos Alexios Aristenos, einen hervorragenden Juristen (vgl. Mortreuil, Histoire du droit Byzantin III 412 ff. 485; C. W. E. Heimbach in der Allgemeinen Encyklopädie von Ersch und Gruber, 1. Sektion, Teil 86 S. 460.389 f.), nicht allein als einen grofsen Rechtsgelehrten, sondern in demselben Mafse auch wegen seiner gediegenen allgemeinen

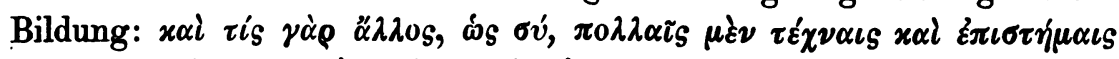

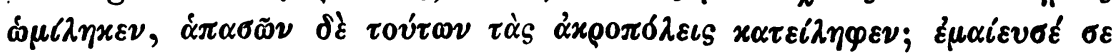

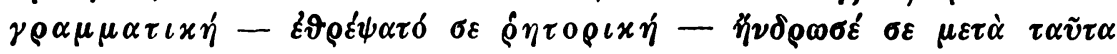

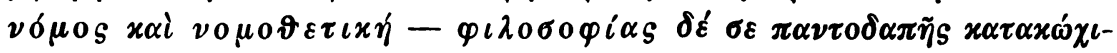
$\mu o v \quad x \tau \lambda$.; vgl. Migne, Patrol. Gr. tom. 133, p. 1243 ss.; ganz ähnlich in einer anderen Rede S. 1273. - Da hätten wir ja wohl einen gebildeten Mann ganz nach dem Herzen unseres Schulmeisters. Allein ich glaube, Aristenos besals eine für seine Zeit aufsergewöhnliche Bildung, und eben deshalb feiert ihn Prodromos; als einen notwendigen Bestandteil der Bildung hat man die Rechtskunde nicht angesehen. Wenn Anna Komnena, welche ihre Alexiade in der Mitte des 12. Jahrhunderts niedergeschrieben, über die Vernachlässigung der höheren Bildung klagt (vgl. ed. Bonn. II 345), so hat sie neben der Grammatik immer nur die Rhetorik und die Philosophie im Auge, nicht das Recht. Es ist vielmehr sicher, dafs es auch im 12. Jahrhundert in Konstanti- 
nopel noch bedeutende Juristen gegeben, ebenso, dafs besondere Rechtsschulen bestanden haben; Heimbach S. 386. Aber das waren eben reine Fachschulen; man konnte sehr wohl ein Gebildeter sein, ohne vom Rechtswesen etwas zu verstehen, und umgekehrt ein recht tüchtiger Rechtsgelehrter, ohne in Rhetorik und Philosophie besonders tief eingedrungen zu sein. Dafür bietet ein anderer Rechtskundiger ein recht

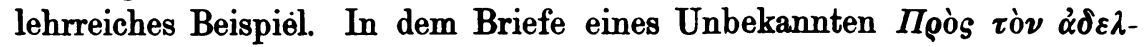

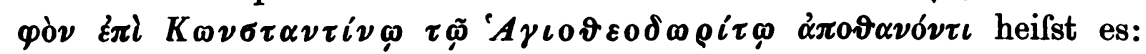

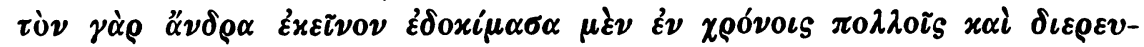

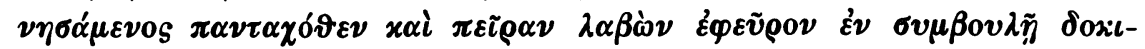

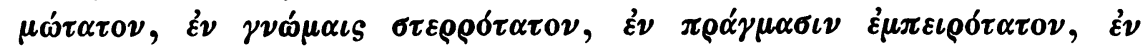

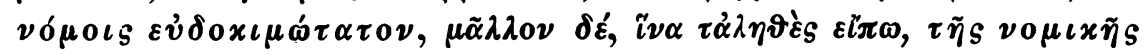

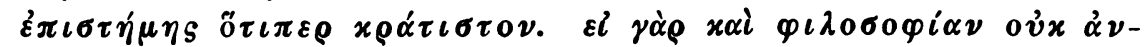

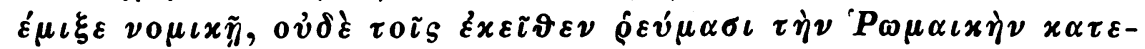

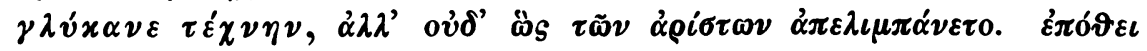

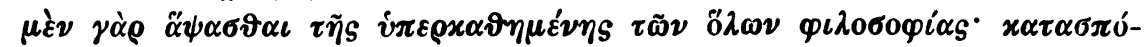

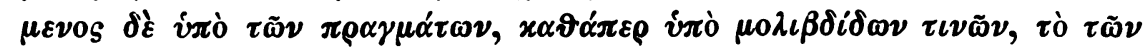

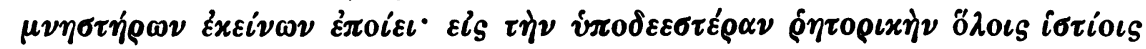

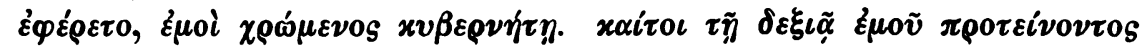

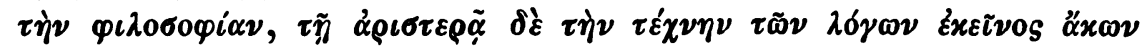

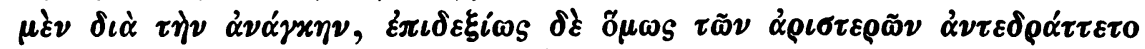

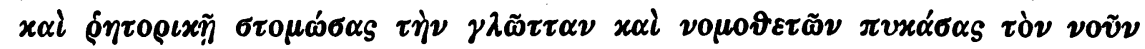

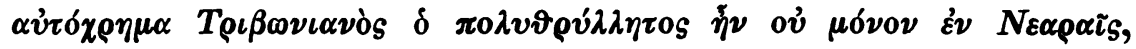

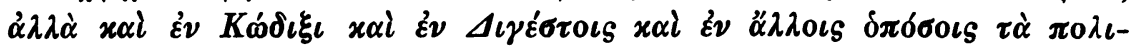

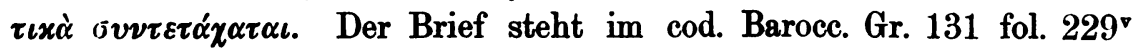
und ist aus $\mathrm{ihm}$, freilich ziemlich inkorrekt, bereits abgedruckt in Fabricii Bibl. Gr. (Hamburg 1724) vol. XII p. 483 s.; aber Fabricius,

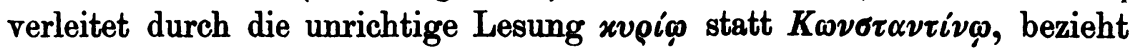
den Brief mit Unrecht auf einen etwas später lebenden Juristen Nikolaos Hagiotheodorites. Es ist aber im Briefe von einem Zeitgenossen des Aristenos die Rede, von demselben Konstantinos Hagiotheodorites, dessen Andenken auch Theodoros Prodromos eine Monodie widmet; vgl. Migne S. 1007. 1008. 1017. 1059 f.

Wenn nun also unser Schulmeister die Philosophie für die $\tau \varepsilon \dot{x} \chi \eta$

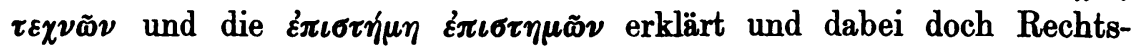
kenntnis als wesentliches Erfordernis der Bildung ansieht, so werden wir mit Notwendigkeit auf die Zeit geführt, in der sich die Rechtskunde neben den sprachlichen und philosophischen Wissenschaften zum letzten Male eine Stellung zu erringen wulste, in welcher man es zum letzten Male für angezeigt hielt, dafs jeder, der sich dem öffentlichen Dienste widmen wollte, eine Unterweisung in der Rechtskunde durch- 
machte: das ist die zweite Hälfte des 11. Jahrhunderts. Man vergleiche darüber die treffliche Darlegung von William Fischer, Studien zur byz. Geschichte des 11. Jahrhunderts, Plauen i. V., 1883, S. 9 ff.

In diese Zeit fällt die Entstehung unseres Schulgespräches, nur aus dem Wissensstande und dem Gedankenkreise dieser Zeit sind die Fragen zu verstehen, welche sich auf das Recht beziehen.

In erster Linie müssen die 60 Bücher der Basilika Gegenstand des Studiums sein. Diese sind in den ersten Jahren der Regierung Leos des Weisen (886-911), wenigstens vor 892 vollendet und veröffentlicht worden; Heimbach S. 313. Doch darf auch die Kenntnis derjenigen Schriften, auf denen die Basilika beruhen, vor allem der Justinianischen Rechtsbücher, nicht vernachlässigt werden: sie werden

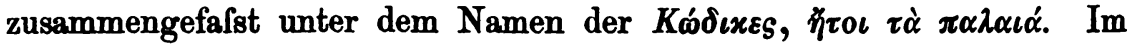

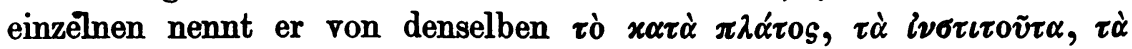

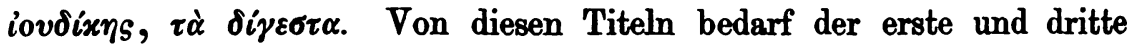

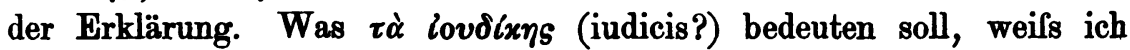

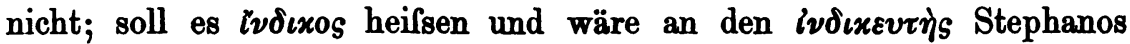
zu denken (Heimbach S. 247)? Ob unter dem Titel $\tau$ ì $x \alpha \tau \dot{\alpha} \pi \lambda \alpha_{\alpha} \tau 0$ S

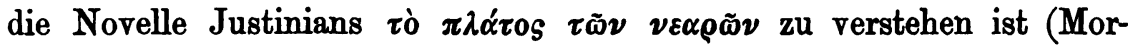
treuil I 34, Heimbach S. 206), oder eines der juristischen Werke des 6. Jahrhunderts (Heimbach S. 302. 256), dürfte schwer zu bestimmen sein. Alle folgenden Fragen versetzen uns mitten ins 11. Jahrhundert. Garidas ist Rechtslehrer, wahrscheinlich an der $1045 \mathrm{zu}$ Konstantinopel errichteten Rechtsschule. Wir besitzen von ihm eine an den Kaiser Konstantinos Dukas (1059-67) gerichteten Bericht über den Unterschied der freiwilligen und unfreiwilligen Totschläge; Mortreuil III 468, Heimbach S. 387. 434. Sodann ist er der Lehrer des Verfassers der

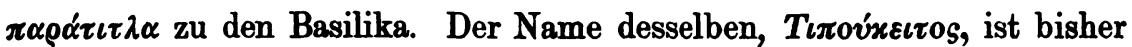
nur aus einer einzigen Handschrift des 14. Jahrhunderts bekannt, von der Leo Allatius eine Abschrift gemacht hat; Mortreuil III 252, Heimbach S. $437 \mathrm{f}$. Der Name ist aus $\tau i$ $\pi 0 \tilde{v}$ $x \varepsilon \tau \tau \alpha \iota$ entstanden. So hätten wir denn in unserer Stelle ein Zeugnis, dafs diese wunderliche Namenbildung bereits im 11. Jahrhundert vollzogen ist. Krumbacher macht mich darauf aufmerksam, dafs eine ähnliche schon viel früher nachzuweisen ist: Von den Deipnosophisten bei Athenaios hat der Rhetor

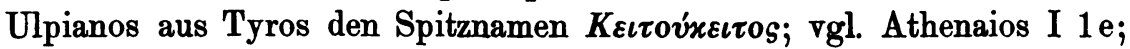

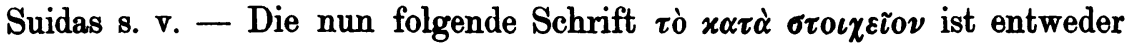

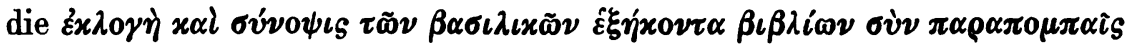

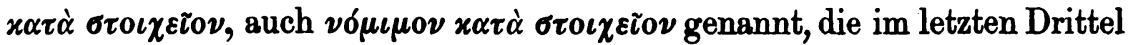
des 11. Jahrhunderts entstand und zum Unterschiede von der erst im 13. Jahrhundert verfalsten synopsis minor auch synopsis maior genannt 
wird; Mortreuil II 435, Heimbach S. 420. Oder es ist noch eine Schrift von Garidas im besonderen angeführt, das $\beta \iota \beta \lambda i o v ~ \pi \varepsilon \rho i ~ \alpha ่ \gamma \omega \gamma \tilde{\nu} \nu$ 'un

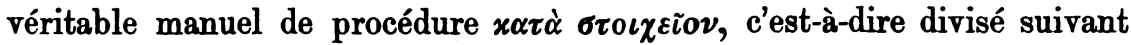
l'ordre des lettres alphabétiques'; Mortreuil III 323, Heimbach S. 387. - Was es mit dem $\delta \delta \eta \gamma \delta$ s für eine Bewandtnis hat, weifs ich nicht. Es dürfte eines der zahlreichen Privatkompendien gewesen sein, welche nicht der Förderung der Wissenschaft, sondern rein praktischen Zwecken dienten; Heimbach S. 425 f. Ein solch offizielles, kaiserlich approbiertes Kompendium aus der Mitte des 11. Jahrhunderts war die $\pi \varepsilon \check{\varrho} \alpha$, eine Sammlung von Beispielen aus der Praxis, an denen die Theorie demonstriert wurde; Mortreuil II 474, Heimbach S. 423, William Fischer S. $55 \mathrm{f}$. Die Beispiele stammen vorwiegend von Eustathios

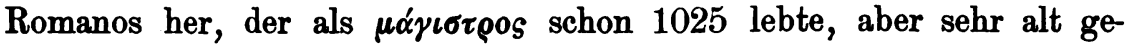
worden zu sein scheint; Heimbach S. 387. Die $\pi \varepsilon\lceil\rho \alpha$ selbst abef ist, wie William Fischer nachweist, von einem anderen erst nach Romanos' Tode herausgegeben worden. $\mathrm{Da}$ ist es denn bemerkenswert, dafs die

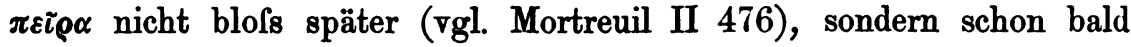

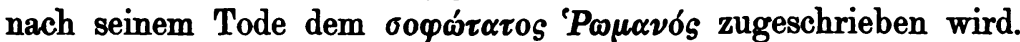

Wie demnach der letzte Teil des Schulgespräches durchaus auf das 11. Jahrhundert hinweist, so ist in dem ganzen übrigen Gespräche nichts enthalten, was dem widerstritte. Dafs neben dem alle Philosophie beherrschenden Aristoteles auch Platon gewürdigt wird, darf uns im Zeitalter des Konstantinos Psellos nicht wundern; vgl. Bruno Rhodius, Beiträge zur Lebensgeschichte und zu den Briefen des Psellos, Plauen i. V., 1892, S. 4. - Die Fragen in der Rhetorik schlielsen sich aufs engste an die fünf uns überlieferten Schriften des Hermogenes an, ohne dals es der Lehrer der Mühe für wert hält ihn namentlich anzuführen. - Was endlich die Fragen in der Grammatik betrifft, so ist es wohl nötig, dals ich die Streitfrage erwähne, welche der von C. Wachsmuth veröffentlichte Teil des Gespräches unter den Fachleuten angeregt hat. Alfred Hilgard, De artis grammaticae a Dionysio Thrace compositae interpretationibus veteribus, Lips. 1880 , S. 7, hält den Zeile 14 erwähnten $\Gamma \varepsilon \dot{\varrho} \rho \gamma \iota o_{s} \tau \iota s$ für denjenigen Georgios, unter dessen Namen im cod. Vatic. Gr. 14 mehrfach Scholien angefuhrt werden; er meint dann, vielleicht wäre es jener Georgios Kurtesios Scholarios, von dem eine grammatische Schrift in einigen Handschriften erhalten sei. Peter Egenolff möchte in seiner Rezension von Hilgards Schrift, Philologische Rundschau II. Jahrg., Bremen 1882, S. 456 f., in

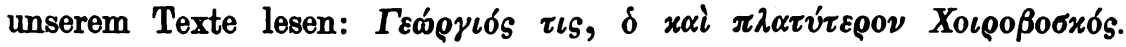
Gustar Uhlig endlich, Dionysii Thracis ars grammatica, Lips. 1883, S. XXXV, verwirft Egenolffs Vorschlag, zweifelt mit Hilgard an der 
Identität von diesem Georgios und Georgios Choiroboskos, lehnt aber auch die Annahme ab, als sei Georgios Kurtesios Scholarios gemeint. Letzteres gewifs mit Recht: Denn dieser Georgios Kurtesios ist unstreitig derselbe, welcher gewöhnlich nur Georgios Scholarios, als Mönch Gennadios genannt wird und Patriarch von Konstantinopel 1454-56

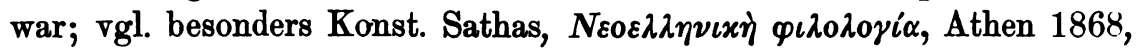

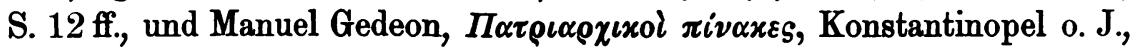
S. $471 \mathrm{ff}$. - Dals in unserem Texte neben Choiroboskos noch ein Georgios angenommen wird, steht fest. Glaubt man ihn -aber fortschaffen zu müssen, so empfiehlt es sich wohl am meisten $\Sigma \dot{\varepsilon} \rho \gamma \iota \delta ́ s$

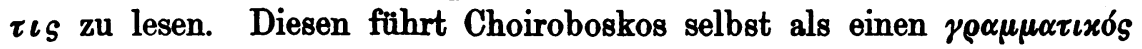
neben Philoponos und Oros an; vgl. Arth. Ludwich, Comm. de Ioanne Philopono grammatico, Regimont. 1888, S. 9 f., und K. Krumbacher, Gesch. d. byz. Litt. S. 277 f. Schreibfehler, oder, wenn man will, Irrtämer, kommen in der Schrift auch sonst vor. So ist wohl Z, 20 statt

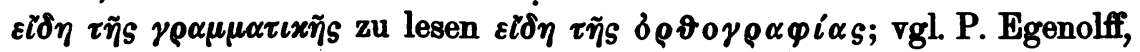
Die orthographischen Stücke der byz. Litt., Heidelberg 1888, S. 4 ff.

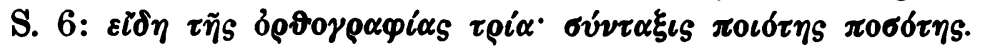

Breslau.

M. Treu. 